\title{
Effect of temperature on tensile properties of injection moulded short glass fibre and glass bead filled ABS hybrids
}

\author{
S. Hashemi* \\ London Metropolitan Polymer Centre, London Metropolitan University, UK
}

Received 24 March 2008; accepted in revised form 30 April 2008

\begin{abstract}
The present study investigated the effect of temperature on tensile strength and modulus of injection moulded ABS polymer reinforced with both short fibres (GF) and spherical glass beads (GB) over the temperature range 25 to $100^{\circ} \mathrm{C}$. Tensile strength, $\sigma_{h}$ and modulus, $E_{h}$, of ABS/GF/GB hybrids increased as volume fraction of the total glass in the hybrids increased. A linear increase in $\sigma_{h}$ and $E_{h}$ was found with increasing the hybrid ratio of the glass fibre, $\chi_{f}$, over the entire temperature range studied. Results indicated that the tensile properties of the ABS/GF/GB hybrid composites at any temperature, $T$, can be estimated from the rule-of-hybrid-mixtures of the form $P_{h}=P_{c f} \chi_{f}+P_{c b}\left(1-\chi_{f}\right)$ where $P_{c f}$ and $P_{c b}$ are the tensile properties of the ABS/GF and ABS/GB composites at temperature $T$, respectively. It was found also that $\sigma_{h}$ and $E_{h}$ both decrease with increasing temperature in a linear manner. The rate at which $\sigma_{h}$ and $E_{h}$ decreased with temperature was dependent upon the hybrid ratio of the glass fibre, $\chi_{f}$, and the total concentration of the glass in the hybrids.
\end{abstract}

Keywords: polymer composites, hybrids, mechanical properties, temperature

\section{Introduction}

It is well recognised that the mechanical properties of polymer composites such as strength and modulus are derived from a combination of the filler and matrix properties and the ability to transfer stresses across the interface between the two constituents. These properties, however, are affected by a number of parameters, such as the concentration of the filler, geometrical shape and the aspect ratio of filler and the degree of interfacial adhesion between the filler and the matrix [1-9]. For example, whist addition of short fibres to polymers matrices enhances strength, addition of spherical shaped particles (e.g. glass beads) cause deterioration. On the other hand, spherical particles with aspect ratio of unity provide isotropic mouldings whereas short fibres having aspect ratio much greater than unity provide anisotropic mouldings. Fibre composites although are strong and stiff when loaded longitudinally (along the length of the fibres) they are quite weak and less stiff when loaded transversely (normal to the fibres).

Hybridisation provides other dimensions to the potential versatility of composite materials. For example, by incorporating glass fibres and glass beads in the same matrix, one may obtain a moulding which may not be as stiff as the fibre reinforced system but stronger than the glass bead system [10, $11]$.

The study by Philips [12] raised the issue surrounding the possible synergistic hybrid effects, in which the properties of the hybrid composite might not follow from a direct consideration of the independent properties of the individual components. A positive or negative hybrid effect was then defined as a positive or negative deviation of a certain mechanical property from the rule-of-mixtures behaviour. In general, tensile strength and modulus of hybrid 
systems do conform to rule of mixtures $[10,11]$. However, the majority of hybrid studies have been conducted at room temperature. Indeed, little is known on the effect of temperature on hybrid properties such as tensile strength and modulus. To this end, this work was undertaken to study the effect of temperature on tensile strength and modulus of ABS polymer filled with short glass fibre and glass bead particles (i.e., ABS/GF/GB hybrid), over the temperature range 25 to $100^{\circ} \mathrm{C}$. To examine the applicability of the rule-of-hybrid-mixtures, tensile properties of $\mathrm{ABS} / \mathrm{GF}$ and $\mathrm{ABS} / \mathrm{GB}$ composites were also studied under the same conditions as the hybrid composites.

\section{Experimental details}

\subsection{Materials}

Owens Corning chopped E-glass fibres (GF) of approximately $6.0 \mathrm{~mm}$ in length and $10 \mu \mathrm{m}$ in diameter and Potters solid spheriglass CP-3000 spherical glass beads (GB) of approximately $12-26 \mu \mathrm{m}$ in diameter were used as reinforcing fillers for Acrylonitrile Butadiene Styrene (ABS) copolymer received by Bayer (Novodut P2H-AT). The ABS and the reinforcing fillers were used to produce a series of $\mathrm{ABS} / \mathrm{GB}, \mathrm{ABS} / \mathrm{GF}$ and $\mathrm{ABS} /$ GB/GF compounds with nominal glass contents of 10,20 and $30 \% \mathrm{w} / \mathrm{w}$.

\subsection{Compounding}

The materials listed in Table 1 were at first mixed to the desired glass content and then dried in an oven at $80^{\circ} \mathrm{C}$ for 4 hours. After drying, each formulation was passed through a Leistritz micro 18GL$7 \mathrm{R}$ co-rotating twin-screw extruder at an average
Table 1. Formulation for composites and hybrids. $\left(\chi_{f}=\right.$ concentration of glass fibres divided by the concentration of total glass)

\begin{tabular}{|c|c|c|c|c|}
\hline \multirow{4}{*}{} & \multicolumn{4}{|c|}{ ABS/Glass Composites } \\
\cline { 2 - 5 } & $\begin{array}{c}\text { Total glass } \\
{[\mathbf{w t} \%]}\end{array}$ & $\begin{array}{c}\text { GF } \\
{[\mathbf{w t} \%]}\end{array}$ & $\begin{array}{c}\text { GB } \\
{[\mathbf{w t} \%]}\end{array}$ & $\begin{array}{c}\text { Hybrid ratio, } \boldsymbol{\chi}_{\mathbf{f}} \\
{[-]}\end{array}$ \\
\hline \multirow{3}{*}{ ABS/GB } & 10 & 0 & 10 & 0 \\
\cline { 2 - 5 } & 20 & 0 & 20 & 0 \\
\cline { 2 - 5 } ABS/GF & 30 & 0 & 30 & 0 \\
\hline & 10 & 10 & 0 & 1 \\
\cline { 2 - 5 } & 20 & 20 & 0 & 1 \\
\cline { 2 - 5 } & 30 & 30 & 0 & 1 \\
\hline \multirow{5}{*}{ Hybrids } & 10 & 5 & 5 & 0.50 \\
\cline { 2 - 5 } & 20 & 5 & 15 & 0.25 \\
\cline { 2 - 5 } & 20 & 10 & 10 & 0.50 \\
\cline { 2 - 5 } & 20 & 5 & 5 & 0.75 \\
\cline { 2 - 5 } & 30 & 20 & 20 & 0.33 \\
\cline { 2 - 5 } & 30 & 15 & 15 & 0.50 \\
\hline
\end{tabular}

screw speed of $60 \mathrm{rpm}$ to produce a homogeneous dispersion of bead and or fibre throughout the matrix. The extruder temperature profile was 203/ $232 / 232 / 235^{\circ} \mathrm{C}$ and the die diameter was $4 \mathrm{~mm}$. The extrudates emerging from the die exit were continuously cooled in a water bath and fed through a granulator to produce pellets for injection moulding process. Pellets were dried in an oven at $80^{\circ} \mathrm{C}$ for 4 hours before being injection moulded into test specimens.

\subsection{Specimen preparation}

BS EN ISO-527 dumbbell shaped tensile bars were produced using a Negri Bossi NB60 injectionmoulding machine at the processing conditions listed in Table 2. The mould used consisted of a single gate cavity producing dumbbell shaped tensile bars of dimensions shown in Figure 1.

Table 2. Injection moulding processing conditions

\begin{tabular}{|c|c|c|c|c|}
\hline Processing condition & $100 \%$ ABS matrix & $\begin{array}{l}\text { Composites \& hybrids } \\
\text { with } 10 \% \text { total filler }\end{array}$ & $\begin{array}{l}\text { Composites \& hybrids } \\
\text { with } 20 \% \text { total filler }\end{array}$ & $\begin{array}{l}\text { Composites \& hybrids } \\
\text { with } 30 \% \text { total filler }\end{array}$ \\
\hline \multicolumn{5}{|l|}{ Barrel temperature $\left[{ }^{\circ} \mathrm{C}\right]$} \\
\hline Zone 1 & 230 & 230 & 230 & 230 \\
\hline Zone 2 & 230 & 230 & 232 & 232 \\
\hline Zone 3 & 232 & 232 & 235 & 235 \\
\hline Mould temperature $\left[{ }^{\circ} \mathrm{C}\right]$ & 70 & 70 & 80 & 80 \\
\hline Injection pressure [MPa] & 8.5 & 8.5 & 9 & 9 \\
\hline Holding pressure [MPa] & 3 & 3 & 3 & 3 \\
\hline Cooling time $[\mathrm{s}]$ & 30 & 30 & 30 & 30 \\
\hline Cycle time [s] & 33 & 33 & 33 & 33 \\
\hline Shot weight $[\mathrm{g}]$ & 27 & 28 & 28 & 29 \\
\hline
\end{tabular}




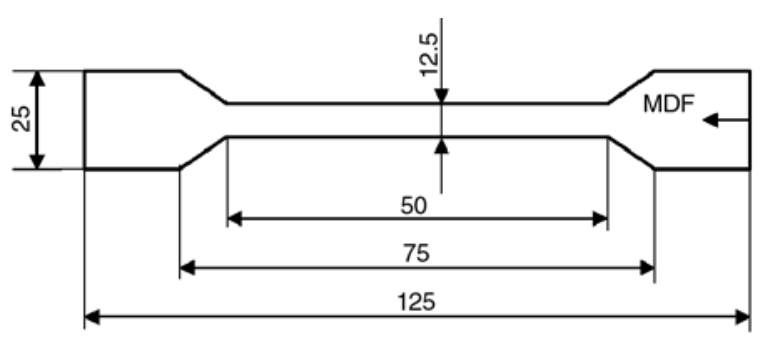

Figure 1. Dimensions of single-gated specimens in millimetres

\subsection{Filler concentration measurements}

The concentration of total glass in each material was determined from the glass residue remained by burning at $550^{\circ} \mathrm{C}$ three weighed samples that were cut from the gauge length of the moulded specimens. After cooling, the ash of glass residue was weighed and the exact weight fraction of glass $\left(w_{g}\right)$ was determined. The $w_{g}$ values were subsequently converted into glass volume fractions $\phi_{g}$ using Equation (1):

$\phi_{g}=\left[1+\frac{\rho_{g}}{\rho_{m}}\left(\frac{1}{w_{g}}-1\right)\right]^{-1}$

Taking the density of the matrix $\left(\rho_{m}\right)$ as $1.12 \mathrm{kgm}^{-3}$ and density of glass $\left(\rho_{g}\right)$ as $2.54 \mathrm{kgm}^{-3}$ gave average glass fibre concentration values of 4.4, 9.5 and $15.5 \% \mathrm{v} / \mathrm{v}$ in GF composites and average glass bead concentration values of 4.2, 9.1 and $14 \% \mathrm{v} / \mathrm{v}$ in GB composite. The volume fraction of glass fibres, $\phi_{f}$, and glass beads, $\phi_{b}$, in each hybrid was calculated from $\phi_{g}$ and the hybrid ratio.

\subsection{Fibre length measurements}

The ash of fibrous material was subsequently spread on a glass slide and placed on the observation stage of a microscope. Magnified fibre images were transmitted to a large screen, and the fibre images were then automatically digitised using Linkam Scientific RTVMS software. From the fibre length distributions, the average fibre length values $\left(L_{f}\right)$ of 550,430 and $360 \mu \mathrm{m}$ were obtained for $4.4,9.5$ and $15.5 \% \mathrm{v} / \mathrm{v}$ glass fibre composites, respectively. Results show decrease in $L_{f}$ with increasing $\phi_{f}$ thus indicating that the average length of the fibre in the moulded specimens was affected by the concentration level of the fibres. The increased damage to fibre length with increasing $\phi_{f}$ was attributed to a greater degree of fibre-fibre interaction and increased in melt viscosity at higher fibre loadings. The latter give rise to higher bending forces on the fibres during compounding and moulding process causing the fibres to break, as noted here.

\subsection{Mechanical testing}

Dumbbell specimens were tested in tension in an Instron 4466 testing machine using pneumatic clamps. Tensile tests were performed at 25, 60 and $100^{\circ} \mathrm{C}$ at a constant crosshead displacement rate of $50 \mathrm{~mm} / \mathrm{min}$. The load-displacement curve for each specimen was recorded using a computer data logger from which tensile modulus and strength were calculated using the initial slope and load at maximum, respectively. For each material at least six specimens were tested at a given temperature. The average tensile strength and modulus values are reported here. The standard deviation for tensile strength and modulus was no more than $2 \mathrm{MPa}$ and $0.08 \mathrm{GPa}$, respectively.

\section{Results and discussions}

\subsection{ABS/GF composites}

The load-extension curves for ABS showed a clear yield point before failure. The incorporation of glass fibres into ABS reduced the overall deformation but enhanced the tensile strength and modulus of the ABS matrix. It must be said, that failure of the ABS/GF composites was brittle over the entire temperature range studied here whereas that of the ABS matrix was ductile. Close examination of the load-extension curves revealed that whilst deformation of the composites was linear at low stresses, it was nonlinear at higher stresses. This observation indicated that interfacial microfailure had occurred around the fibres in the composites during tensile loading.

The effect of fibre concentration, $\phi_{f}$, on tensile modulus of $\mathrm{ABS} / \mathrm{GF}$ composites, $E_{c f}$, at 25, 60 and $100^{\circ} \mathrm{C}$ is shown in Figure 2 where it can be seen that at a any given temperature $E_{c f}$ increases linearly with increasing $\phi_{f}$. The linear dependence between $E_{c f}$ and $\phi_{f}$ is consistent with the modified 'rule-of-mixtures' given by Equation (2): 


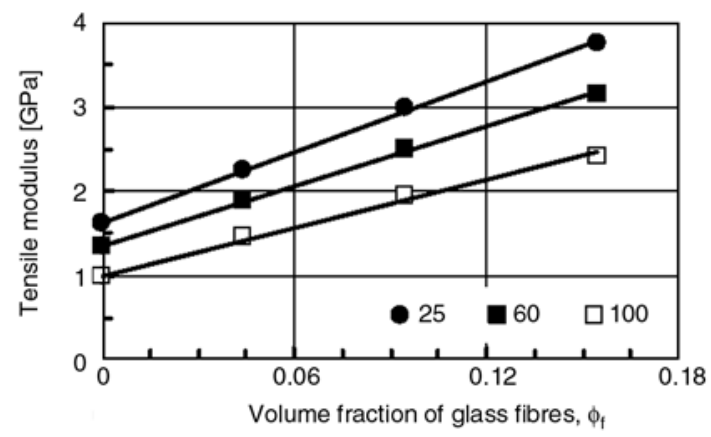

Figure 2. Tensile modulus of ABS/GF composite, $E_{c f}$, versus volume fraction of glass fibres, $\phi_{f}$, at 25,60 and $100^{\circ} \mathrm{C}$

$$
E_{c f}=E_{m}+\left(\lambda_{1} \lambda_{2} E_{f}-E_{m}\right) \phi_{f}
$$

where $E_{c f}$ and $E_{m}$ are the tensile modulus values of $\mathrm{ABS} / \mathrm{GF}$ and the ABS matrix at temperature $T$ and $E_{f}$ is the modulus of the glass fibres taken in this study as $75 \mathrm{GPa}$ and assumed to be independent of temperature. $\lambda_{1}$ and $\lambda_{2}$ are, respectively, the fibre length and fibre orientation factors for tensile modulus, taking into account fibre misalignment and fibre length variation in the composite. Equation (2) can alternatively be written in the form of Equation (3):

$E_{c f}=E_{m}+\left(\lambda_{E} E_{f}-E_{m}\right) \phi_{f}$

where $\lambda_{E}=\lambda_{1} \lambda_{2}$ is termed the overall fibre efficiency factor for composite modulus. According to Equation (3), value of $\lambda_{E}$ at any given temperature can be determined from the slope of the line $E_{c f}$ versus $\phi_{f}$. Using the slope of the linear regression lines in Figure 2, one obtains the variation in $\lambda_{E}$ with temperature as depicted in Figure 3. As can be seen, over the temperature range studied here, $\lambda_{E}$ is much less than one and decreases with increasing

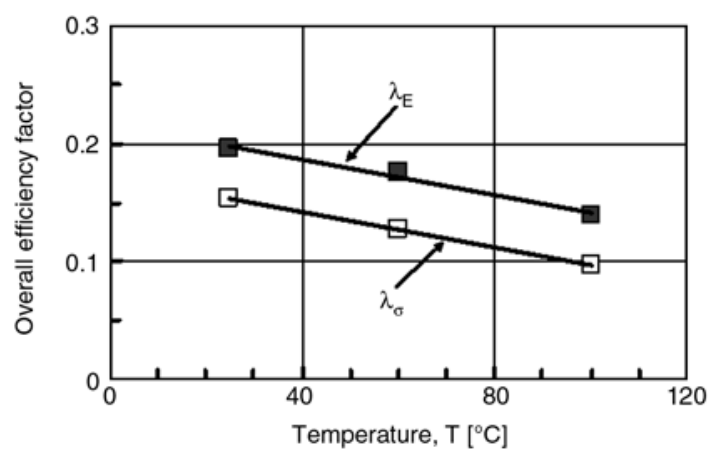

Figure 3. Overall efficiency factors for tensile modulus, $\lambda_{E}$, and tensile strength, $\lambda_{\sigma}$, of ABS/GF composites versus temperature, $T$

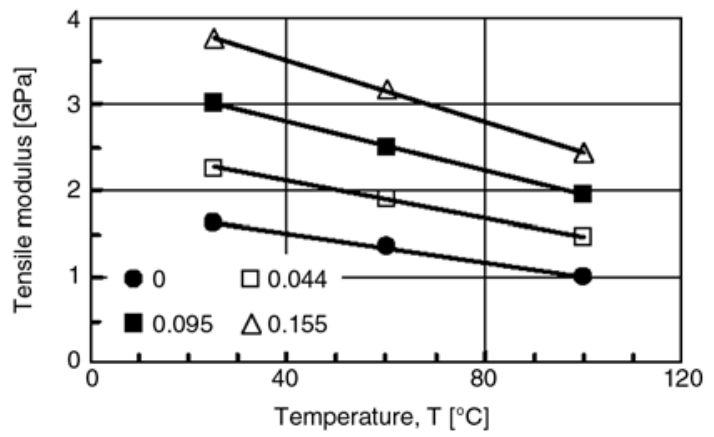

Figure 4. Tensile modulus of ABS/GF composite, $E_{c f}$ versus temperature, $T$, for fibre volume fractions, $\phi_{f}$, of $0,0.044,0.095$ and 0.155

temperature in a linear manner. The linear dependence in Figure 3 can be expressed by Equation (4):

$\lambda_{E}=0.217-7.63 \cdot 10^{-4} \mathrm{~T}$

The effect of temperature on tensile modulus of ABS/GF composites is more explicitly shown in Figure 4 where it can be seen that $E_{c f}$ decreases linearly with increasing temperature. It can be seen also that the rate of decrease as given by the slope of the lines in Figure 4 (i.e. $\mathrm{d} E_{c f} / \mathrm{d} T$ ) depends on the volume fraction of glass fibres in the composites. As shown in Figure 5, when $\mathrm{d} E_{c f} / \mathrm{d} T$ values are plotted against $\phi_{f}$, one obtains a linear dependence given by Equation (5):

$\frac{\mathrm{d} E_{c f}}{\mathrm{~d} T}=-\left(0.0083+0.060 \phi_{f}\right)$

According to Cox shear lag model [13], fibre length efficiency factor $\lambda_{1}$ can be evaluated from the relationship given by Equation (6):

$\lambda_{1}=1-\frac{\tanh \beta}{\beta}$

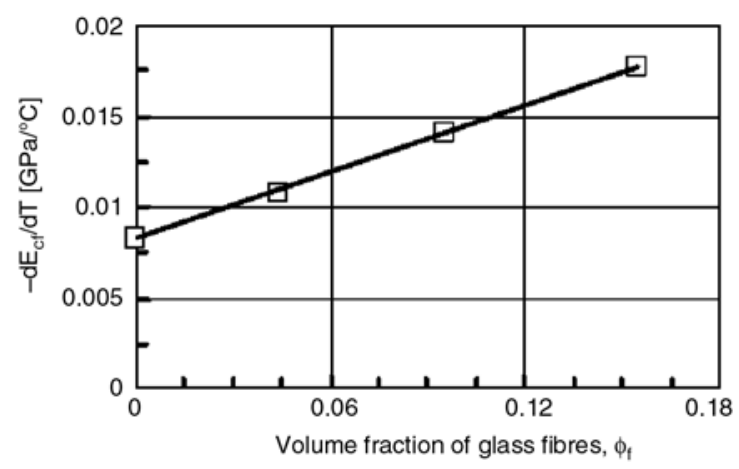

Figure 5. Effect of $\phi_{f}$ on the slope of the line $E_{c f}$ versus temperature in Figure 4 
where $\beta$ is defined by Equation (7):

$$
\beta=\frac{\bar{L}_{f}}{2}\left(\frac{4 E_{m}}{E_{f} d^{2} \ln \varphi}\right)^{\frac{1}{2}}
$$

where $d$ is the diameter of the fibres. For square packing arrangement of fibres, $\varphi$ can be obtained from the relationship given by Equation (8):

$$
\varphi=\sqrt{\frac{\pi}{4 \phi_{f}}}
$$

Values of $\lambda_{1}$ calculated via Equation (6) are presented in Table 3 where it can be seen that $\lambda_{1}$ decreases with increasing temperature but shows no significant variation with respect to $\phi_{f}$. Also given in Table 3 are values of $\lambda_{2}$ obtained from the ratio $\lambda_{E} / \lambda_{1}$ and as can be seen $\lambda_{2}$ decreases with increasing temperature and increases slightly with increasing $\phi_{f}$.

The effect of fibre volume fraction on tensile strength of ABS/GF composites, $\sigma_{c f}$, at 25,60 and $100^{\circ} \mathrm{C}$ is shown in Figure 6 where it can be seen that over the entire temperature range studied here, $\sigma_{c f}$ increases with increasing fibre concentration in a nonlinear manner showing tendency towards levelling off or reaching a maximum on approaching fibre concentration value of approximately $16 \% \mathrm{v} / \mathrm{v}$. The levelling off effect (or reaching a maximum)

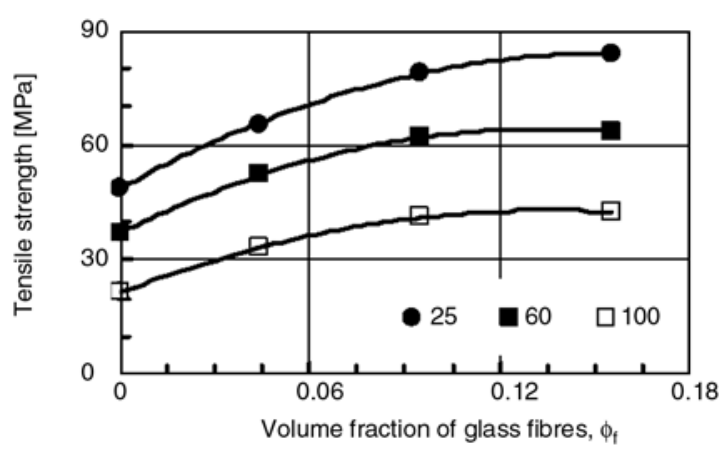

Figure 6. Tensile strength of ABS/GF composite, $\sigma_{c f}$, versus volume fraction of glass fibres, $\phi_{f}$, at 25,60 and $100^{\circ} \mathrm{C}$ which is also noted in several other injection moulded glass reinforced polymer systems $[3,4,8$, 9] is mainly attributed to the separation distance between the fibres becoming sufficiently small as to restrict the flow of the matrix material between fibres. This effect and the higher stress concentration in the matrix due to the greater number of fibre ends at high $\phi_{f}$, reduces the gain in strength which one would expect otherwise.

The nonlinear rise in $\sigma_{c f}$ with increasing $\phi_{f}$ as depicted in Figure 6 can be described by a second order polynomial function of the form given by Equation (9):

$$
\sigma_{c}=a_{0}+a_{1} \phi_{f}+a_{2} \phi_{f}^{2}
$$

However, as illustrated in Figure 7, for fibre concentration values in the range 0 to $10 \% \mathrm{v} / \mathrm{v}$, variation of $\sigma_{c f}$ with $\phi_{f}$ is reasonably linear with regression coefficients of greater than 0.97 . Within this $\phi_{f}$ range, variation of $\sigma_{c f}$ with $\phi_{f}$ can be described by the modified 'rule-of- mixtures' for tensile strengths as given by Equation (10):

$\sigma_{c f}=\sigma_{m}+\left(\lambda_{3} \lambda_{4} \sigma_{f}-\sigma_{m}\right) \phi_{f}$

where $\sigma_{f}$ is tensile strength of the glass fibres taken in this study as $2470 \mathrm{MPa}$ and $\sigma_{m}$ is the tensile strength of the ABS matrix. $\lambda_{3}$ and $\lambda_{4}$ are respectively, the fibre length and orientation factors for

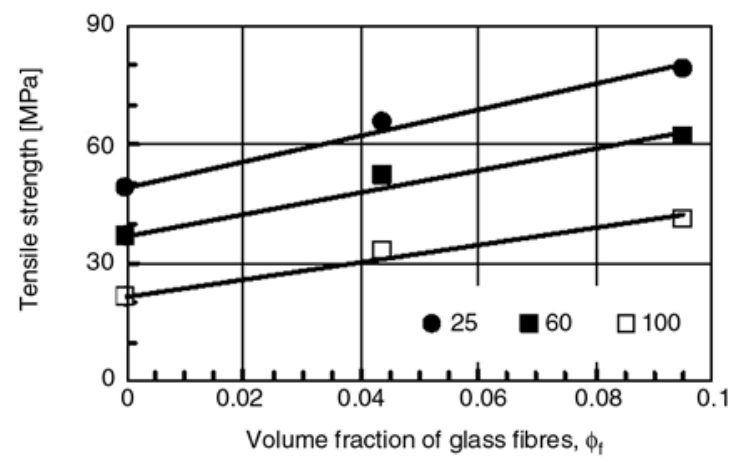

Figure 7. Tensile strength of ABS/GF composite, $\sigma_{c f}$, versus volume fraction of glass fibres, $\phi_{f}$, at 25,60 and $100^{\circ} \mathrm{C}$ for $\phi_{f}$ values in the range 0 to

\begin{tabular}{|c|c|c|c|c|c|c|c|c|c|}
\hline \multirow{2}{*}{$\phi_{\mathrm{f}}$} & \multicolumn{3}{|c|}{$\lambda_{\mathrm{E}}$} & \multicolumn{3}{|c|}{$\lambda_{1}$} & \multicolumn{3}{|c|}{$\lambda_{2}$} \\
\hline & $25^{\circ} \mathrm{C}$ & $60^{\circ} \mathrm{C}$ & $100^{\circ} \mathrm{C}$ & $25^{\circ} \mathrm{C}$ & $60^{\circ} \mathrm{C}$ & $100^{\circ} \mathrm{C}$ & $25^{\circ} \mathrm{C}$ & $60^{\circ} \mathrm{C}$ & $100^{\circ} \mathrm{C}$ \\
\hline 0.044 & 0.196 & 0.175 & 0.139 & 0.827 & 0.811 & 0.780 & 0.237 & 0.216 & 0178 \\
\hline 0.095 & 0.196 & 0.175 & 0.139 & 0.811 & 0.793 & 0.760 & 0.242 & 0.221 & 0.183 \\
\hline 0.155 & 0.196 & 0.175 & 0.139 & 0.802 & 0.783 & 0.748 & 0.244 & 0.223 & 0.186 \\
\hline
\end{tabular}
$10 \% \mathrm{v} / \mathrm{v}$

Table 3. Fibre efficiency parameters for tensile modulus of ABS/GF composites 
Table 4. Fibre efficiency parameters for composite strength as a function of fibre volume fraction at 25,60 , and $100^{\circ} \mathrm{C}$

\begin{tabular}{|c|c|c|c|c|c|c|c|c|c|}
\hline \multirow{2}{*}{$\phi_{\mathrm{f}}$} & \multicolumn{3}{|c|}{$\lambda_{\sigma}$} & \multicolumn{3}{|c|}{$\lambda_{4} \approx \lambda_{2}$} & \multicolumn{3}{|c|}{$\lambda_{3}$} \\
\hline & $25^{\circ} \mathrm{C}$ & $60^{\circ} \mathrm{C}$ & $100^{\circ} \mathrm{C}$ & $25^{\circ} \mathrm{C}$ & $60^{\circ} \mathrm{C}$ & $100^{\circ} \mathrm{C}$ & $25^{\circ} \mathrm{C}$ & $60^{\circ} \mathrm{C}$ & $100^{\circ} \mathrm{C}$ \\
\hline 0.044 & 0.153 & 0.127 & 0.097 & 0.237 & 0.216 & 0178 & 0.646 & 0.588 & 0.545 \\
\hline 0.095 & 0.153 & 0.127 & 0.097 & 0.242 & 0.221 & 0.183 & 0.632 & 0.575 & 0.530 \\
\hline 0.155 & 0.153 & 0.127 & 0.097 & 0.244 & 0.223 & 0.186 & 0.627 & 0.570 & 0.522 \\
\hline
\end{tabular}

tensile strength, which take into account fibre length variation and misalignment.

Equation (10) can alternatively be written in the form of Equation (11):

$\sigma_{c f}=\sigma_{m}+\left(\lambda_{\sigma} \sigma_{f}-\sigma_{m}\right) \phi_{f}$

where $\lambda_{\sigma}=\lambda_{3} \lambda_{4}$ is the overall efficiency factor for tensile strength whose value at any given temperature can be determined from the slope of the line $\sigma_{c f}$ versus $\phi_{f}$ in Figure 7. The effect of temperature on $\lambda_{\sigma}$ is also shown in Figure 3 for comparative purposes where it can be seen that $\lambda_{\sigma}$ is lower than $\lambda_{E}$ and decreases likewise with increasing temperature. The linear dependence between $\lambda_{\sigma}$ and temperature as shown in Figure 3 can be expressed by Equation (12):

$\lambda_{\sigma}=0.172-7.52 \cdot 10^{-4} T$

Assuming $\lambda_{4} \approx \lambda_{2}$, values of $\lambda_{3}$ for composite strength was evaluated as a function of temperature $\left(\lambda 3=\lambda_{\sigma} / \lambda_{2}\right)$. It can be seen from Table 4 , that $\lambda_{3}$ for composite strength like $\lambda_{1}$ for composite modulus decreases with increasing temperature. It is also notable that $\lambda_{4}$ is consistently lower than $\lambda_{1}$ thus indicating that tensile strength is more affected by the shortness of the fibres than tensile modulus.

Using the values $\lambda_{3}$ given in Table 4 , the critical fibre lengths, $L_{c}$ was calculated for each composite as a function of temperature using the Kelly-Tyson relationship given by Equation (13) [14]:

$$
L_{c}=\frac{L_{f}}{2 \lambda_{3}}
$$

Results obtained from this analysis are presented in Table 5 where it can be seen that $L_{c}$ increases with increasing temperature but decreases with increasing $\phi_{f}$ mainly due to the reduction in the average length of the fibre, $L_{f}$, with increasing $\phi_{f}$.

The effect of temperature on $\sigma_{c f}$ is more explicitly shown in Figure 8. As can be seen, for a fixed $\phi_{f}$, tensile strength of the composite, $\sigma_{c f}$, decreases lin-
Table 5. Fibre critical length as a function of fibre volume fraction at 25,60 and $100^{\circ} \mathrm{C}$

\begin{tabular}{|c|c|c|c|}
\hline \multirow{2}{*}{$\boldsymbol{\phi}_{\mathbf{r}}$} & \multicolumn{3}{|c|}{$\mathbf{L}_{\mathbf{c}}[\boldsymbol{\mu} \mathbf{m}]$} \\
\cline { 2 - 4 } & $\mathbf{2 5}^{\circ} \mathbf{C}$ & $\mathbf{6 0}^{\circ} \mathbf{C}$ & $\mathbf{1 0 0}^{\circ} \mathbf{C}$ \\
\hline 0.044 & 426 & 468 & 505 \\
\hline 0.095 & 340 & 374 & 406 \\
\hline 0.155 & 287 & 316 & 345 \\
\hline
\end{tabular}

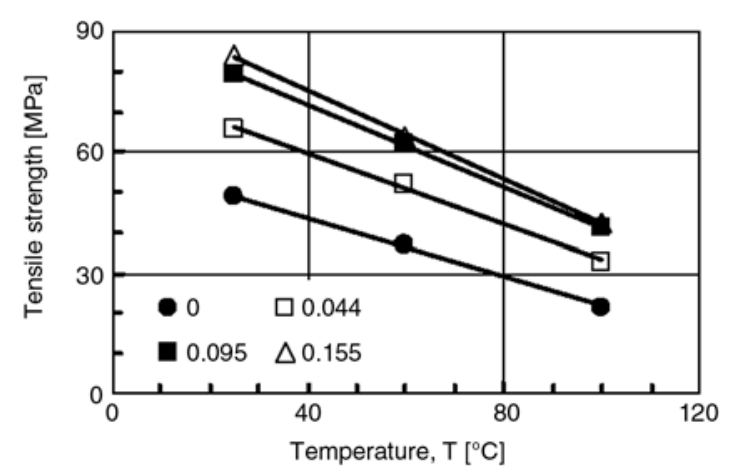

Figure 8. Tensile strength of ABS/GF composite, $\sigma_{c f}$, versus temperature, $T$, for fibre volume fractions, $\phi_{f}$, of $0,0.044,0.095$ and 0.155

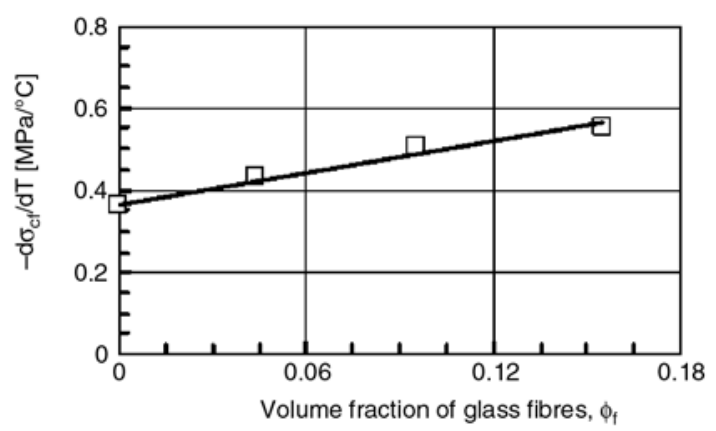

Figure 9. Effect of $\phi_{f}$ on the slope of the line, $\sigma_{c f}$, versus temperature in Figure 8

early with increasing temperature. As illustrated in Figure 9, when slope of the lines in Figure 8 (i.e. $\left.\mathrm{d} \sigma_{c f} / \mathrm{d} T\right)$ are plotted versus $\phi_{f}$, one obtains a linear dependence which can be reasonably expressed by Equation (14):

$$
\frac{\mathrm{d} \sigma_{c f}}{\mathrm{~d} T}=-\left(3.64+1.305 \phi_{f}\right)
$$

Comparing Equation (5) and Equation (14) reveals that for the same volume fraction of fibres, com- 
posite strength is more affected by the change in temperature than composite modulus.

\subsection{ABS/GB composites}

The incorporation of glass beads into ABS reduced the tensile strength, but increased the tensile modulus, of the ABS matrix. Close examination of the load-extension curves once again indicated that whilst deformation of the composites was linear at low stresses, it was nonlinear at higher stresses. This observation suggested that interfacial microfailure also occurred around the glass bead particles during loading of the ABS/GB composites.

The effect of the volume fraction of glass beads, $\phi_{b}$, on tensile modulus of $\mathrm{ABS} / \mathrm{GB}$ composites, $E_{c b}$, at 25, 60 and $100^{\circ} \mathrm{C}$ is shown in Figure 10. It can be observed that $E_{c b}$ increases with increasing $\phi_{b}$ in a linear manner. The linear dependence between $E_{c b}$ and $\phi_{b}$ follows the Einstein [15] relationship given by Equation (15):

$$
E_{c b}=E_{m}\left(1+k_{E} \phi_{b}\right)
$$

where $E_{m}$ is modulus of the matrix and $k_{E}$ is the Einstein coefficient having a value of 2.5 for spherical shaped fillers. The slope of the linear regression lines in Figure 10 suggests $k_{E}$ values of 2.55, 2.38 and 2.31 at 25,60 and $100^{\circ} \mathrm{C}$, respectively for the $\mathrm{ABS} / \mathrm{GB}$ system.

It is worth noting that at any given temperature as shown in Figure 11, gain in modulus due to addition of short glass fibres is much greater than that due to addition of glass beads. Also, the rate at which slope of the lines in Figures 2 and 10 changes with temperature, is a linear and decreasing function of temperature. The linear dependence

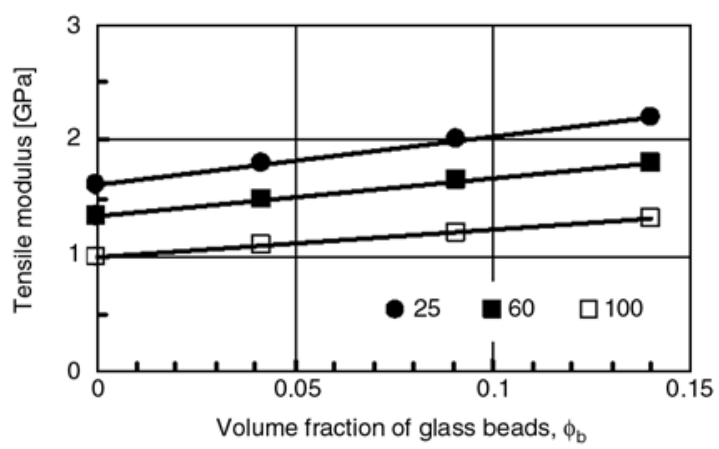

Figure 10. Tensile modulus of ABS/GB composite, $E_{c b}$, versus volume fraction of glass beads, $\phi_{b}$, at 25,60 and $100^{\circ} \mathrm{C}$

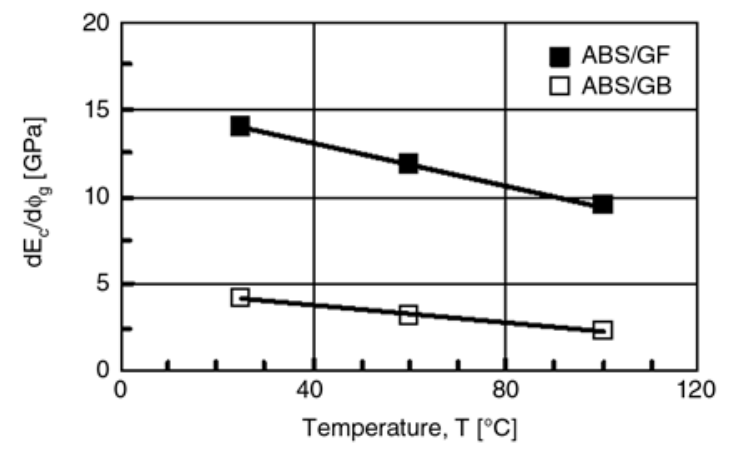

Figure 11. Effect of temperature on the slope of the lines in Figures 2 and 10

for both composite systems can be expressed by Equations (16) and (17);

$$
\begin{aligned}
& \frac{\mathrm{d} E_{c f}}{\mathrm{~d} \phi_{f}}=15.47-0.061 T \\
& \frac{\mathrm{d} E_{c b}}{\mathrm{~d} \phi_{b}}=4.711-0.024 T
\end{aligned}
$$

The above equations reveal that at any given temperature, tensile modulus of the ABS/GF composite is always greater than that of $\mathrm{ABS} / \mathrm{GB}$ counterpart. Also, modulus of the composite material is more affected by the change in volume fraction of glass fibres than that of glass beads.

The effect of temperature on tensile modulus of $\mathrm{ABS} / \mathrm{GB}$ composites, $E_{c b}$, is more explicitly shown in Figure 12 where it can be seen that $E_{c b}$ decreases linearly with increasing temperature. It can be seen also that the rate of decrease as given by the slope of the lines in Figure 12 (i.e. $\mathrm{d} E_{c b} / \mathrm{d} T$ ) depends on the volume fraction of glass beads in the composite. As shown in Figure 13, when $\mathrm{d} E_{c b} / \mathrm{d} T$ values are plotted against $\phi_{b}$, a linear dependence is obtained

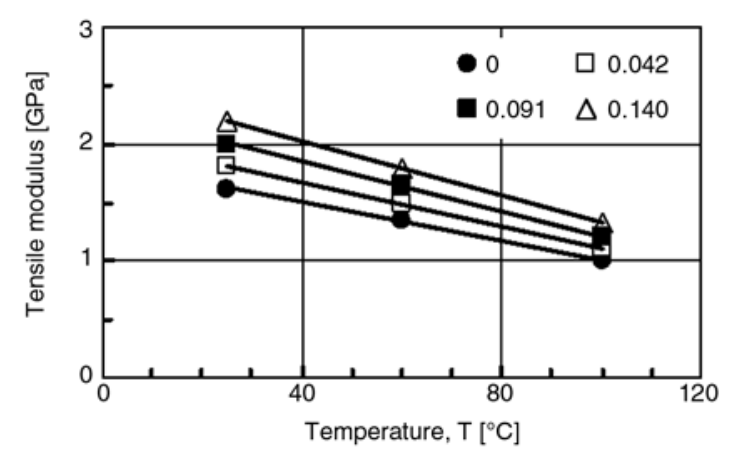

Figure 12. Tensile modulus of ABS/GB composite, $E_{c b}$, versus temperature, $T$, for glass bead volume fractions, $\phi_{b}$, of $0,0.042,0.091$ and 0.140 


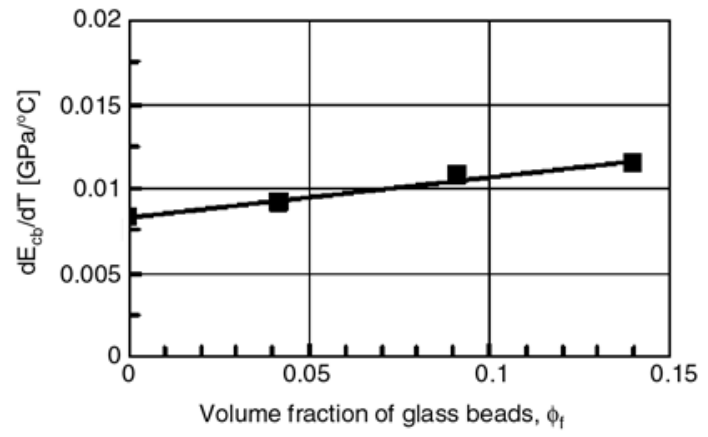

Figure 13. Effect of $\phi_{b}$ on the slope of the line, $\sigma_{c b}$, versus temperature in Figure 11

which can be reasonably expressed by Equation (18):

$$
\frac{\mathrm{d} E_{c b}}{\mathrm{~d} T}=-\left(0.0083+0.024 \phi_{b}\right)
$$

The effect of $\phi_{b}$ on tensile strength of ABS/GB composites, $\sigma_{c b}$, at 25,60 and $100^{\circ} \mathrm{C}$ is shown in Figure 14 . As can be seen, $\sigma_{c b}$ decreases linearly with increasing $\phi_{b}$, over the entire temperature range studied here. It is worth noting that temperature has no significant influence upon the slope of the lines in Figure 14.

The linear dependence between $\sigma_{c b}$ and $\phi_{b}$ as depicted in Figure 14 follows Piggott and Leidner [16] relationship given by Equation (19):

$\sigma_{c b}=K \sigma_{m}-b \phi_{b}$

where $K$ is a stress concentration factor and $b$ is a constant whose value depends upon particle-matrix adhesion. Fitting the best regression lines to the data in Figure 14 gives $K$ values of near unity $(0.99)$ and $b$ values in the range 32.48 to 34.01 .

The effect of temperature on tensile strength of $\mathrm{ABS} / \mathrm{GB}$ composites $\sigma_{c b}$, is more explicitly shown

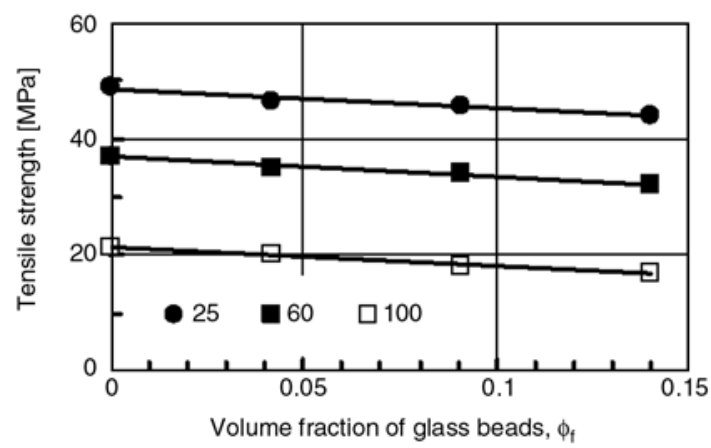

Figure 14. Tensile strength of ABS/GB composite, $\sigma_{c b}$, versus volume fraction of glass beads, $\phi_{b}$, at 25,60 and $100^{\circ} \mathrm{C}$

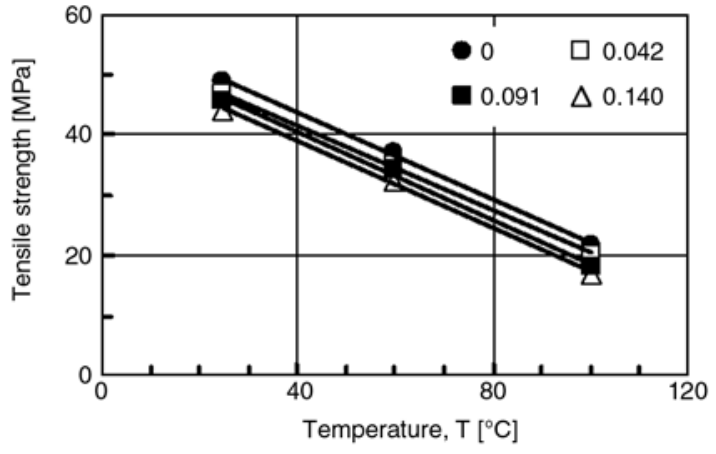

Figure 15. Tensile strength of ABS/GB composite, $\sigma_{c b}$, versus temperature, $T$, for glass bead volume fractions, $\phi_{b}$, of 0, 0.042, 0.091 and 0.140

in Figure 15. It can be seen that $\phi_{b}$ has no significant effect upon the slope of the lines in Figure 15, thus indicating that the rate of change of $\sigma_{c b}$ with temperature is independent of the glass bead concentration. Results suggest the rate of change follows Equation (20):

$$
\frac{\mathrm{d} \sigma_{c b}}{\mathrm{~d} T}=-0.364
$$

\subsection{ABS/GF/GB hybrids}

The effect of hybridisation on tensile modulus and strength was studied here in terms of hybrid ratio, $\chi_{f}$, is defined by Equation (21):

$\chi_{f}=\frac{\phi_{f}}{\phi_{g}}$

where $\phi_{f}$ is the volume fraction of the fibres and $\phi_{g}$ is the total volume fraction of glass, in the hybrid.

Figure 16 show the effect of $\chi_{f}$ on tensile modulus of ABS/GF/GB hybrids, $E_{h}$, at 25,60 and $100^{\circ} \mathrm{C}$ for total glass concentration values of 10,20 and $30 \%$ w/w, respectively. It can be seen that $E_{h}$ increases with increasing $\chi_{f}$ in a linear manner. Results also reveal that at a fixed temperature, the effect of increasing $\phi_{g}$ is an upwards shift in the modulus values coupled with an increase in the slope of the lines $E_{h}$ versus $\chi_{\mathrm{f}}$. The linearity of $E_{h}$ with $\chi_{f}$ as depicted in Figure 16a-16c suggests hybrid tensile modulus conforms to the rule-ofmixtures given by Equation (22):

$$
E_{h=} E_{c f} \chi_{f}+E_{c b}\left(1-\chi_{f}\right)
$$



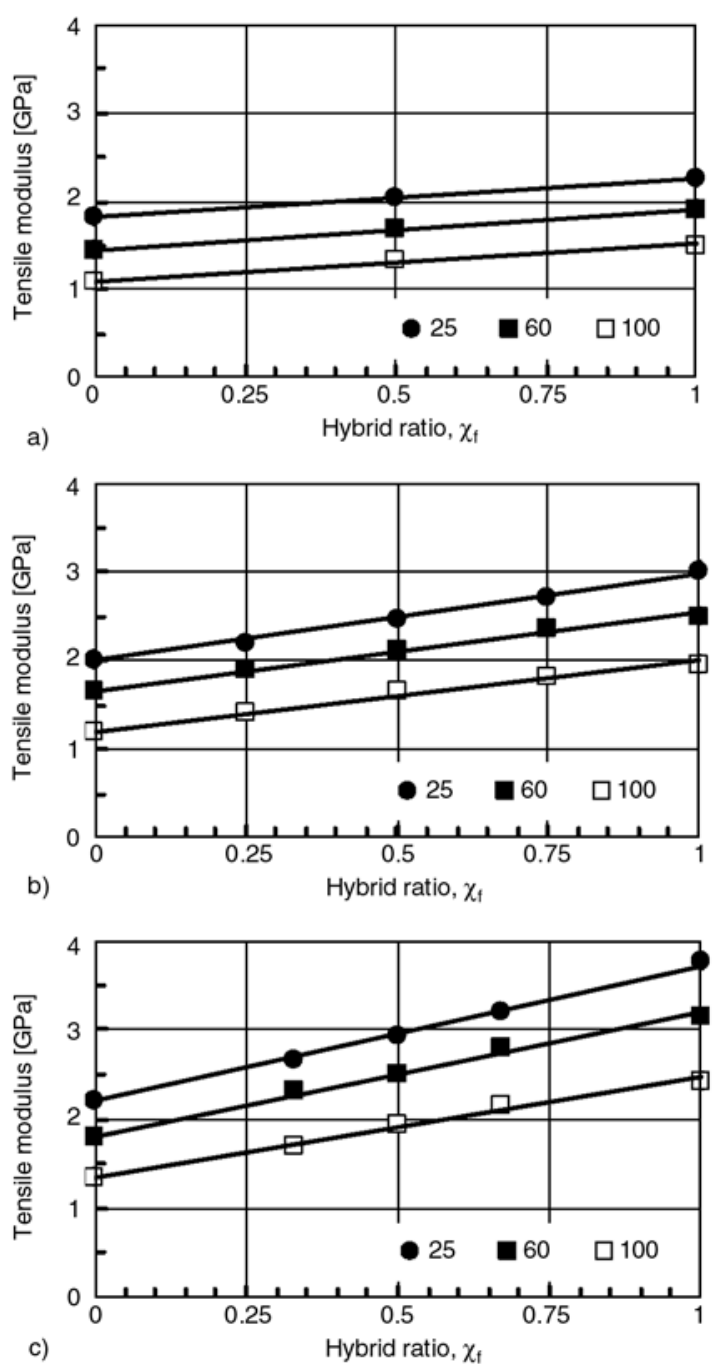

Figure 16. Tensile modulus of ABS/GF/GB hybrids, $E_{h}$, versus hybrid ratio, $\chi_{f}$, at 25,60 and $100^{\circ} \mathrm{C}$ for total glass concentration values of; (a) $10 \%$, (b) $20 \%$ and (c) $30 \%$ by weight

where $E_{c f}$ and $E_{c b}$ are the modulus values of $\mathrm{ABS} / \mathrm{GF}$ and $\mathrm{ABS} / \mathrm{GB}$ composites respectively, as defined by Equations (2) and (15). The lines fitted through the data in Figure 16 are according to Equation (22) where it can be seen that rule-ofmixtures for hybrids describes the data extremely well.

The effect of temperature on tensile modulus of ABS/GF/GB hybrids is shown in Figure 17 where it can be seen that $E_{h}$ decreases with increasing temperature. However, the rate at which $E_{h}$ decreases with temperature appears to be more affected by the total concentration of the glass in the hybrid than by the hybrid ratio, $\chi_{f}$. The effect of increasing, $\chi_{f}$, is simply an upward shift in $E_{h}$ values. It is also evident that $\chi_{f}$ has no significant
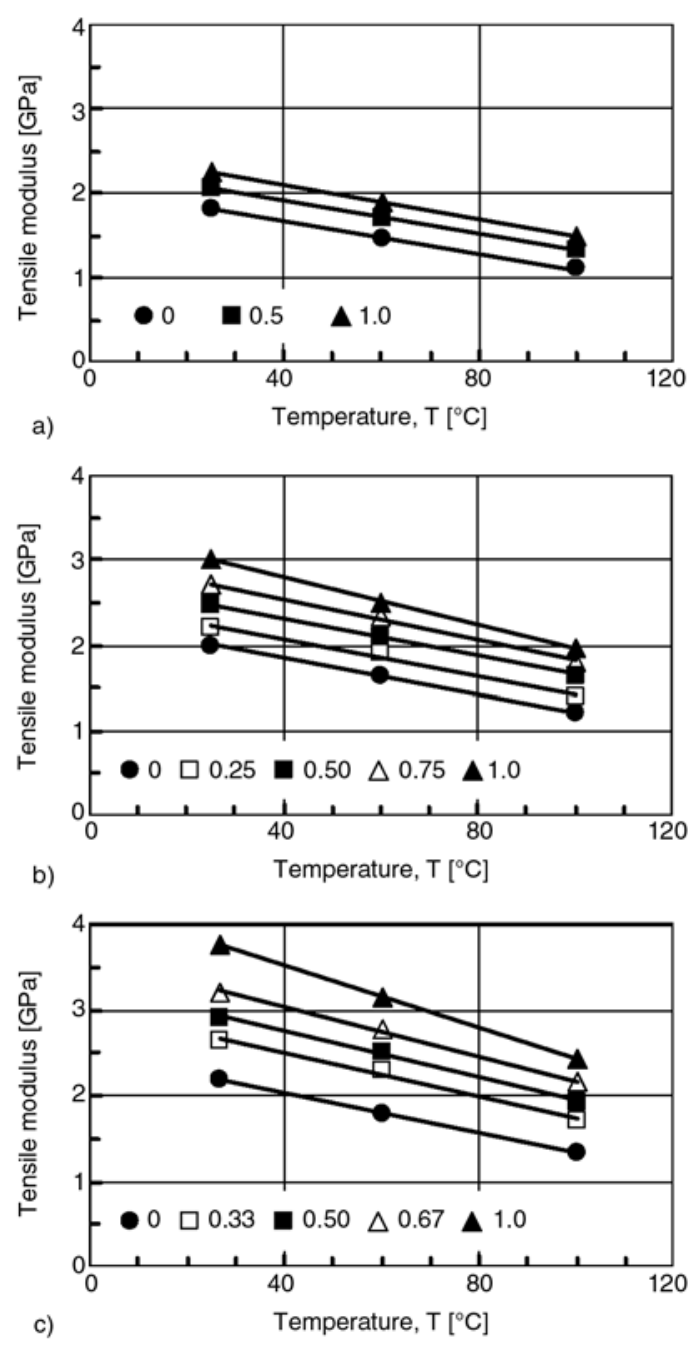

Figure 17. Tensile modulus of ABS/GF/GB hybrid, $E_{h}$, versus temperature, $T$, for various hybrid ratios, $\chi_{f}$, and the total glass concentration values of; (a) $10 \%$, (b) $20 \%$ and (c) $30 \%$ by weight

effect upon the slope of the lines in Figure 17, particularly at low glass concentration values.

The effect of $\chi_{f}$ on tensile strength of ABS/GF/GB hybrids, $\sigma_{h}$, at 25,60 and $100^{\circ} \mathrm{C}$ for total glass content values of 10, 20 and 30\% w/w is shown in Figure 18. Results show that $\sigma_{h}$ increases with increasing $\chi_{f}$ in a linear manner thus indicating that the hybrid tensile strength like tensile modulus follows rule-of-mixtures given by Equation (23):

$\sigma_{h}=\sigma_{c f} \chi_{f}+\sigma_{c b}\left(1-\chi_{f}\right)$

where $\sigma_{c f}$ and $\sigma_{c b}$ are the tensile strengths of $\mathrm{ABS} / \mathrm{GF}$ and $\mathrm{ABS} / \mathrm{GB}$ composites respectively, as defined by Equations (9) and (19). The rate at which $\sigma_{h}$ increases with increasing $\chi_{f}$ (i.e., $\mathrm{d} \sigma_{h} / \mathrm{d} \chi_{f}$ ) is shown in Figure 19 as a function of temperature 

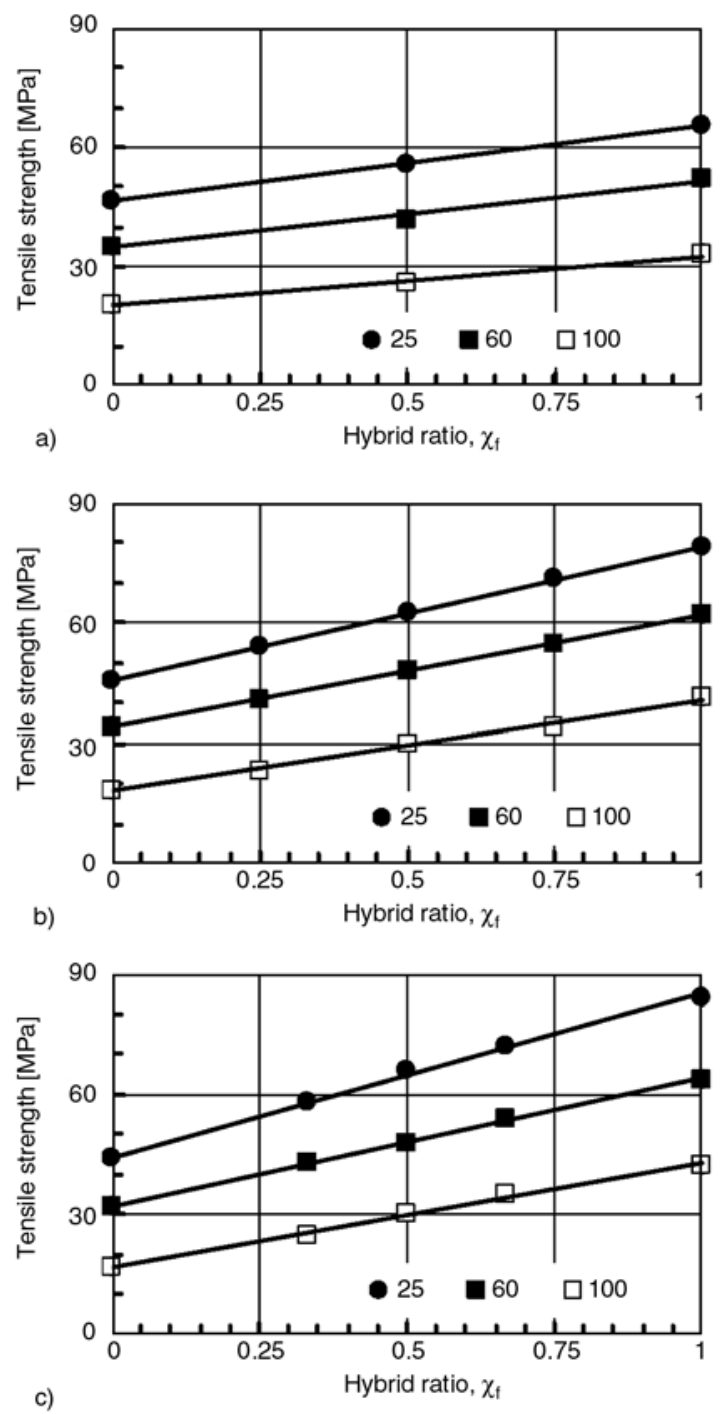

Figure 18. Tensile strength of ABS/GF/GB hybrids, $\sigma_{h}$, versus hybrid ratio, $\chi_{f}$, at 25,60 and $100^{\circ} \mathrm{C}$ for the total glass concentration values of; (a) $10 \%$, (b) $20 \%$ and (c) $30 \%$ by weight

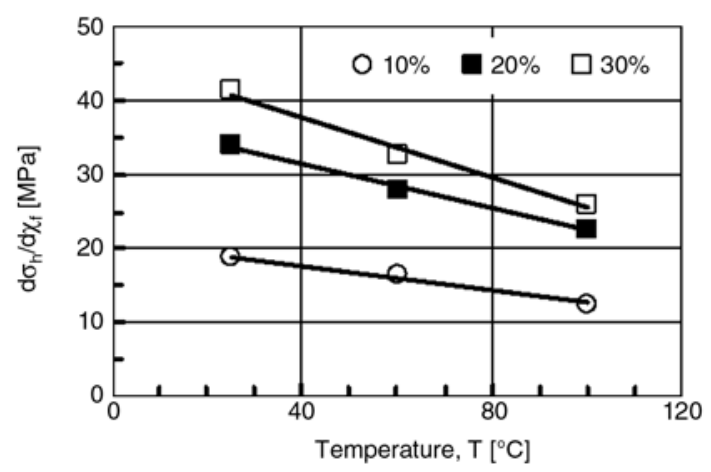

Figure 19. Effect of temperature on the slope of the line $\sigma_{h}$ versus $\chi_{f}$ for total glass concentration values of 10,20 and $30 \%$ wt

for the total glass weight percentage values of 10 , 20 and $30 \%$. Results show that $\mathrm{d} \sigma_{h} / \mathrm{d} \chi_{f}$ decreases

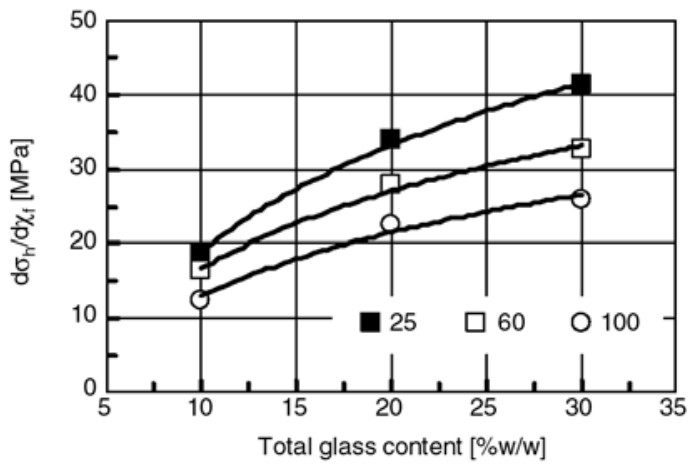

Figure 20. Effect of glass content on the slope of the line $\sigma_{h}$ versus $\chi_{f}$ at 25,60 and $100^{\circ} \mathrm{C}$
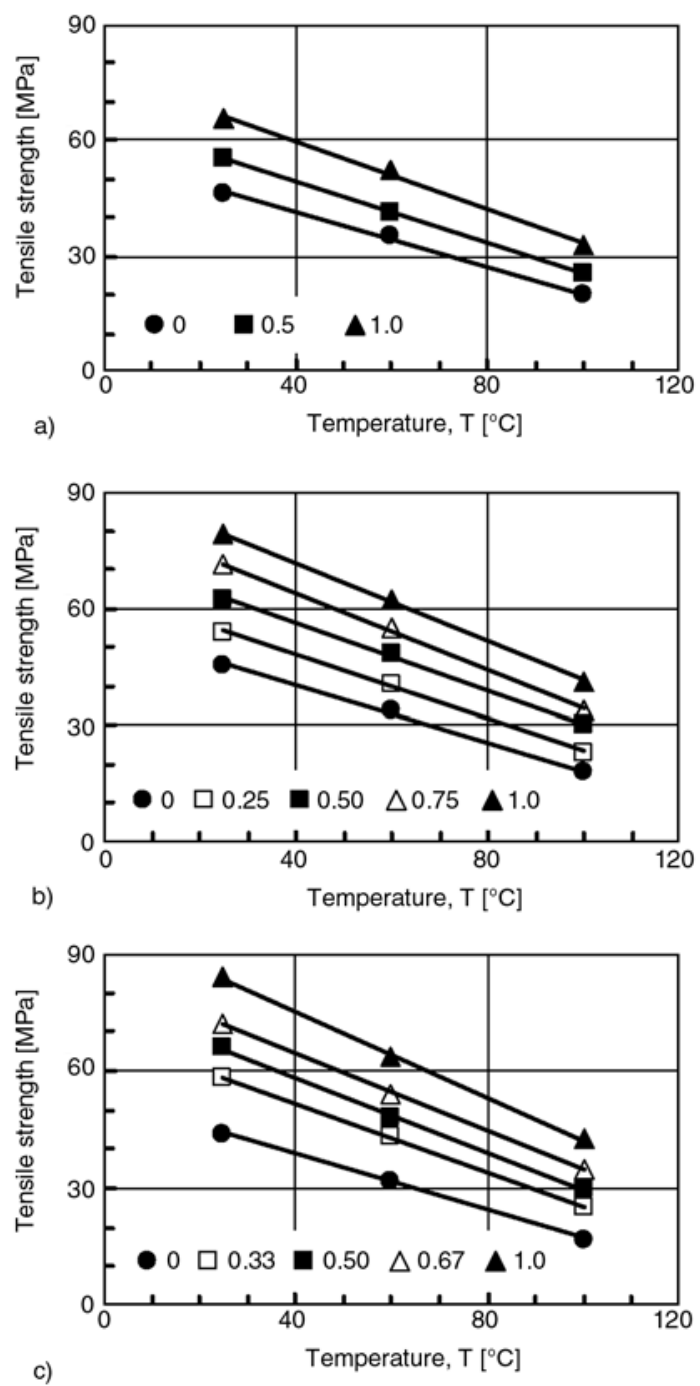

Figure 21. Tensile strength of ABS/GF/GB hybrid, $\sigma_{h}$, versus temperature, $T$, for various hybrid ratios, $\chi_{f}$, and total glass concentration values of;

(a) $10 \%$, (b) $20 \%$ and (c) $30 \%$ by weight

linearly with increasing temperature. However, as shown in Figure 20, the effect of temperature becomes more significant as weight percentage of the total glass in the hybrid is increased. 


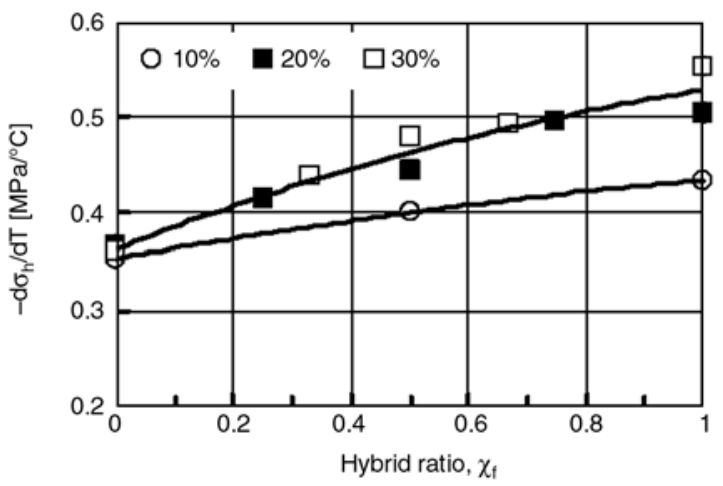

Figure 22. Effect of hybrid ratio, $\chi_{f}$, on the slope of the lines $\sigma_{h}$ versus temperature, $T$, in Figure 21 for total glass concentration values of; (a) $10 \%$, (b) $20 \%$ and (c) $30 \%$ by weight

The effect of temperature on $\sigma_{h}$ is shown more explicitly in Figure 21. Results show that $\sigma_{h}$ decreases with increasing temperature in a linear manner. The rate at which $\sigma_{h}$ decreases with increasing temperature (i.e. $\mathrm{d} \sigma_{h} / \mathrm{d} T$ ) as a function of hybrid ratio, $\chi_{f}$, is shown in Figure 22 where it can be seen $\mathrm{d} \sigma_{h} / \mathrm{d} T$ increases with increasing $\chi_{f}$ and the total glass content in the hybrid. However, as illustrated in Figure 22, when the glass content in the hybrid is high, variation of $\mathrm{d} \sigma_{h} / \mathrm{d} T$ with $\chi_{f}$ becomes almost independent of the glass content.

\section{Conclusions}

A positive and negative hybrid effect in hybrid composites is defined as positive or negative deviation of a certain mechanical property from the ruleof-mixtures. It was found;

(i) Hybrid tensile strength, $\sigma_{h}$, and modulus, $E_{h}$, both increase with increasing the hybrid ratio of the glass fibre, $\chi_{f}$ in a linear manner. The variation of $E_{h}$ and $\sigma_{h}$ with $\chi_{f}$ obeys rule-of-mixtures.

(ii) Hybrid tensile strength, $\sigma_{h}$, and modulus, $E_{h}$, both increase with increasing the total concentration of the glass in the hybrid.

(iii) Hybrid tensile strength and modulus both decrease with increasing temperature in a linear manner.

\section{References}

[1] Hashemi S., Gilbride M. T., Hodgkinson J. M.: Mechanical property relationships in glass-filled polyoxymethylene. Journal of Materials Science, 31, 5017-5025 (1996).
[2] Nabi Z. U., Hashemi S.: Influence of short glass fibres and weldlines on the mechanical properties of injection-moulded acrylonitrile-styrene-acrylate copolymer. Journal of Materials Science, 33, 2985-3000 (1998).

[3] Hashemi S.: Influence of temperature on weldline strength of injection moulded short glass fibre styrene maleic anhydride polymer composites. Journal of Plastics, Rubber and Composites, 31, 318-324 (2002).

[4] Hashemi S.: Thermal effects on weld and unweld tensile properties of injection moulded short glass fibre reinforced ABS composites. Express Polymer Letters, 1, 688-697 (2007).

[5] Necar M., Irfan-ul-Haq M., Khan Z.: Temperature and weld-line effects on mechanical properties of CPVC. Journal of Materials Processing Technology, 142, 247-255 (2003).

[6] Fu S-Y., Lauke B., Mäder E., Yue C-Y., Hu X.: Tensile properties of short-glass-fiber- and short-carbonfiber-reinforced polypropylene composites. Composites, Part A: Applied Science and Manufacturing, 31, 1117-1125 (2000).

[7] Fisa B.: Mechanical degradation of glass fibres during compounding with polypropylene. Polymer Composites, 6, 232-241 (1985).

[8] Thomason J. L.: Micromechanical parameters from macromechanical measurements on glass reinforced polypropylene. Composites Science and Technology, 62, 1455-1468 (2002).

[9] Thomason J. L.: Micromechanical parameters from macromechanical measurements on glass reinforced polyamide 6,6. Composites Science and Technology, 61, 2007-2016 (2001).

[10] Yilmazer U.: Tensile, flexural and impact properties of a thermoplastic matrix reinforced by glass fiber and glass bead hybrids. Composites Science and Technology, 44, 119-125 (1992).

[11] Hashemi S., Elmes P., Sandford S.: Hybrid effects on mechanical properties of polyoxymethylene. Polymer Engineering and Science, 37, 45-58 (1997).

[12] Phillips L. N.: The hybrid effect - Does it exist? Composites, 7, 7-8 (1976).

[13] Cox H. L.: The elasticity of strength of paper and other fibrous materials. British Journal of Applied Physics, 3, 72-79 (1952).

[14] Kelly A., Tyson W. R.: Tensile properties of fibrereinforced metals: Copper/tungsten and copper/ molybdenum. Journal Mechanics Physics of Solids, 13, 329-350 (1965).

[15] Einstein A.: Eine neue Bestimmung der Moleküldimensionen. Annalen der Physik, 19, 289-306 (1906).

[16] Leidner J., Woodhams R. T.: The strength of polymeric composites containing spherical fillers. Journal of Applied Polymer Science, 18, 1639-1654 (1974). 